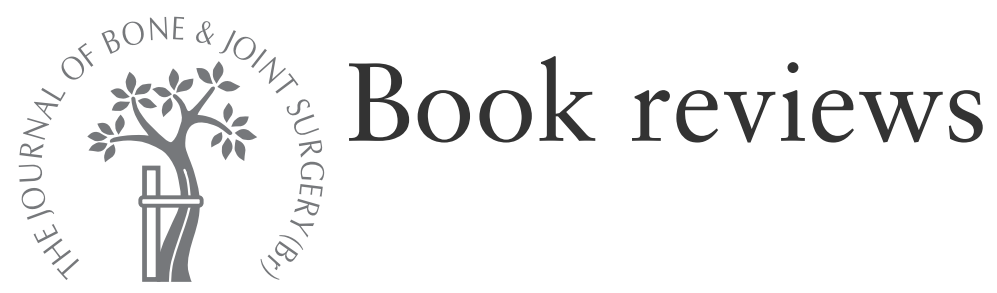

(C)British Editorial Society of Bone and Joint Surgery

doi:10.1302/0301-620X.90B8. 21132 \$2.00 J Bone Joint Surg [Br] 2008;90-B:1119-20.

\section{Advanced Reconstruction: Shoulder} Edited by J. D. Zuckerman

Pp. 679. Rosemont: American Academy of Orthopaedic Surgeons, 2007. ISBN: 13:978-0-89203-392-8. £143.95.

This is a glossy, colourful surgical recipe book of a publication, incorporating extensive and comprehensive cover of both trauma and elective surgery. It is a first edition from the American Academy which states that it "is not intended to present the only, or necessarily the best methods or procedures ... but rather is intended to represent an approach, view, statement of opinion of the author(s) or producer(s), which may be helpful to others who face similar situations." I feel it achieves this aim well.

The majority of contributors are well published authors from North America with a smaller representation from Europe. Each author follows a pleasing and easy to follow format which neatly complements operative planning. It includes indications, contraindications, alternative treatments, results (usually with a tabulated summary of published literature), techniques, post-operative regimens and how to avoid pitfalls and complications. There is a refreshing approach to explain some finer points of surgical technique which are often overlooked in textbooks; the latter two sections are useful and interesting if sometimes only as reassurance. Each chapter ends by tabulating the relevant ICD-9 codes.

The Instability and Chronic Dislocation chapters cover a wide range of clinical scenarios and include excellent comparisons between open and arthroscopic techniques. Although they are mentioned as potential options for bone grafting the anterior glenoid, the one omission in this otherwise comprehensive review is a detailed description of the Bristow and Latarjet techniques.

The cover of Management of Rotator Cuff Tears is a little light and has a strong inclination towards arthroscopic techniques. This probably reflects the widespread adoption of such surgery for those conditions by many surgeons. It does not reflect the enormous number of commercially-available instruments, anchors and fixation systems currently on the market. In an area of some controversy amongst shoulder surgeons, it does well to concentrate on principles of surgical technique and has a particularly good piece on 'soft tissue mobilisation of rotator cuff tears'. Similarly the chapter on Management of Acromioclavicular and Sternoclavicular Joint Injuries does not discuss the newer artificial ligament systems and rather concentrates on surgical principles.

The cover of fracture management of the proximal humerus, clavicle and scapula is rich. This reflects the recent return to favour of operative intervention and a claim is staked for all surgical options: locking plates and screws, percutaneous techniques, intramedullary devices (clavicle included) and prosthetic replacement.

The Management of Neurologic Deficits/Muscle Weakness is an interesting chapter describing established options for these difficult but uncommon problems. Arthroscopic and open techniques for the Management of Frozen Shoulder are eloquently described by leading exponents of each option.

Finally the chapter addressing Glenohumeral Arthritis gives a comprehensive appraisal of a large array of options for both primary and revision surgery. Rheumatoid disease and cuff tear arthropathy are given special consideration and surgical techniques for stemmed prostheses, glenoid components, resurfacing implants and the reverse geometry shoulder are all carefully described.

This is not an essential book for a general orthopaedic surgeon doing the occasional shoulder operation but should be carefully considered by the shoulder specialist who values the opinions of others and appreciates how subtle tips and tricks may improve their own practice.

Mark A. A. Crowther

\section{Advanced Reconstruction: Elbow \\ Edited by K. Yamaguchi, G. J. W. King, M.D. McKee, S. W. M. O'Driscoll \\ Pp. 470. Rosemont: American Academy of Orthopaedic Surgeons, 2007. ISBN: 13:9780892033911. £143.95.}

Published by the American Academy to partner its shoulder equivalent, this is also a colourful, user-friendly guide to operative surgery from an eminent field of North American authors and a few European counterparts. The layout is the same as the shoulder book and the many positive points do not need to be reiterated. The book has four sections (Arthroscopy; Soft-Tissue and Sports Injuries; Arthroplasty; Posttraumatic Reconstruction) which combine to cover elbow surgery extensively and comprehensively.

The Arthroscopy section starts with invaluable advice about basic set-up and equipment and progresses through a series of chapters describing a wide variety of techniques, including reduction and internal fixation of fractures. Some of the chapters are punchy descriptions full of useful technical tips. The second section on Soft tissue and Sports Injuries includes the common procedures for common conditions, such as tennis elbow and ulnar nerve compression, but also advanced techniques of ligament reconstruction and mosaicplasty for capitellar osteochondritis dissecans. 
The section on Arthroplasty includes a description of hemiarthroplasty, six different total elbow arthroplasties, radial head replacement and cover of complications including those to the soft tissues, infection and peri-prosthetic fracture. Each chapter is written by a leading exponent and comprises an unsurpassed collection of surgical experience and advice. The final section deals with a variety of complex challenging conditions likely to fall within the remit of a specialist elbow surgeon, including non- and malunions, proximal radio-ulnar synostosis, arthrodesis, the flail elbow and osteoarticular allografts.
The numerous arthroscopic, intra-operative and radiological images have been carefully selected to complement the text and are often supplemented by colour photography or black and white line drawings. These are all extremely useful and are a major factor in making this a very good reference book for the dedicated elbow surgeon. The summaries of published surgical outcomes relevant to each chapter are appropriate in this age of evidence-based medicine. It complements the classic textbooks on the subject.

Mark A. A. Crowther 